\title{
Exercícios físicos como ferramenta de enfrentamento às comorbidades associadas à obesidade: revisão da literatura
}

\author{
Physical exercise as a coping to the comorbidities associated with obesity tool: \\ literature review
}

\section{El ejercicio físico como una herramienta para hacer frente a las comorbilidades asociadas a la obesidad: revisión de la literatura}

\author{
Gleice Eugenia da Silva e SOUZA $\mathbf{A}^{\mathbf{1}}$ \\ Miguel Ribeiro PRUDENCIATTO ${ }^{1}$ \\ Rubens Shigueyasu TANAKA ${ }^{1}$ \\ Anderson MARTELLI ${ }^{2}$ \\ Lucas Risseti DELBIM ${ }^{3}$ \\ ${ }^{1}$ Graduação em Educação Física pela Faculdade Mogiana do Estado de São Paulo, FMG, Mogi Guaçu - SP, Brasil \\ ${ }^{2}$ Mestrando em Ciências Biomédicas - Uniararas; \\ Especialista em Laboratório Clínico - Patologia Clínica pela Faculdade de Ciências Médicas - UNICAMP. \\ Professor da Faculdade Mogiana do Estado de São Paulo, FMG, Mogi Guaçu - SP, Brasil \\ ${ }^{3}$ Mestre em Sustentabilidade e Qualidade de Vida - (UNIFAE). \\ Docente do Curso de Educação Física da Faculdade Mogiana do Estado de São Paulo, FMG, Mogi Guaçu - SP, Brasil
}

\begin{abstract}
Resumo
A obesidade é caracterizada pelo acúmulo excessivo de gordura corporal no indivíduo, sendo um fator de risco para uma série de doenças. A presente revisão apresenta como objetivo abordar os aspectos epidemiológicos do sobrepeso e da obesidade em adultos, como importante fator de agravo no âmbito da saúde coletiva. A revisão foi realizada a partir de pesquisa bibliográfica em literatura especializada e livros acadêmicos orientando-se pela busca de contribuições oriundas da prática do exercício físico sistematizado enquanto ferramenta de enfrentamento aos deletérios efeitos orgânicos emancipados pelo excesso de gordura corporal. Os benefícios desta prática sistematizada são consensuais, como melhorias no condicionamento cardiovascular, no controle do diabetes mellitus, no aspecto psicológico e social e contribuem positivamente para a melhoria da qualidade de vida. No entanto para que a prática de exercícios seja efetiva, há a necessidade de acompanhamento por profissionais devidamente credenciados.
\end{abstract}

Descritores: Obesidade; Saúde Pública; Exercício.

\begin{abstract}
Obesity is characterized by excessive accumulation of body fat in an individual, being a risk factor for a number of diseases. This review presents how to approach the epidemiology of overweight and obesity in adults, as an important factor grievance under the collective health. The survey was conducted from a literature review of the literature, consulted with scientific articles and additionally consulting academic books being guided by the search for contributions arising from the practice of systematic physical exercise as a tool for addressing the deleterious effects emancipated by excess organic of body fat. The benefits of this systematic practice are consensual as improvements in cardiovascular fitness, control of diabetes mellitus, psychological and social conditions and contribute positively to improving the quality of life. However for the practical exercises to be effective, there is need for prescription and monitoring by professionals duly accredited.
\end{abstract}

Descriptors: Obesity; Public Health; Exercise.

\begin{abstract}
Resumen
La obesidad se caracteriza por la acumulación excesiva de grasa corporal en el individuo, siendo un factor de riesgo para una serie de enfermedades. Esta revisión presenta el objetivo de acercarse a los aspectos epidemiológicos de sobrepeso y obesidad en adultos, como un factor agravante importante en el contexto de la salud pública. La encuesta fue realizada a partir de una revisión bibliográfica de la literatura, y consultó artículos científicos y, además de los libros de texto de consulta guiada por la búsqueda de las contribuciones de la práctica de ejercicios físicos como hacer frente herramienta para los efectos fisiológicos deletéreos emancipados por exceso de la grasa corporal. Los beneficios de esta práctica sistemática están de acuerdo en que las mejoras en la condición cardiovascular, el control de la diabetes mellitus, las enfermedades psicológicas y sociales y contribuyen positivamente a la mejora de la calidad de vida. Sin embargo, para los ejercicios prácticos que sean eficaces, es necesario para la prescripción y supervisión por profesionales debidamente acreditados.
\end{abstract}

Descriptores: Obesidad; Salud Pública; Ejercicio. 


\section{INTRODUÇÃO}

A obesidade pode ser definida como o fator de risco do novo milênio ${ }^{1}$ e faz parte do grupo de doenças crônicas não transmissíveis (DCNT) caracterizando-se pelo acúmulo excessivo de gordura corporal que acarreta prejuízos à saúde dos indivíduos $^{2}$. A etiologia da obesidade é multifatorial que envolve aspectos ambientais e genéticos, sendo caracterizada como um problema de saúde pública mundial, tanto nos países desenvolvidos quanto nos países em desenvolvimento que apresentam elevação de sua prevalência ${ }^{3}$.

Está amplamente demonstrado na literatura científica que a obesidade apresenta grande associação com inúmeros males à saúde como a hipertensão arterial sistêmica (HAS), doença cardíaca coronariana, diabetes tipo II, dislipidemias, entre outros ${ }^{4-9}$.

De acordo com a Organização Mundial de Saúde (OMS) 1,4 bilhões de adultos, em nível mundial, apresentam excesso de peso, dos quais pelo menos 500 milhões são clinicamente obesos. Reforçando ainda, o sobrepeso e a obesidade vêm sendo consideradas epidemias de proporções mundiais - pandemias, tendo sido reconhecidos como transtornos à saúde pública ${ }^{10}$.

Matsudo et al. ${ }^{11}$ afirmam que um dos fatores responsáveis pela maior prevalência da obesidade é, sem dúvida, o sedentarismo ou a insuficiente prática de exercícios físicos (EF) regulares. Segundo a OMS, esta insuficiência ou este processo de inatividade física colocam o ser humano mais próximo de seu declínio existencial ${ }^{10}$.

Em relatório recente foi revelado que a inatividade física é o quarto fator de risco isolado de óbito no mundo, e que o número expressivo de mortes chega a mais de 3 milhões de pessoas por ano. Consta ainda neste informe que o estilo de vida hipocinético é um fator de risco para as $\mathrm{DCNT}^{10}$. Desta forma o enfrentamento ao sedentarismo figura como um procedimento de essencial importância em propostas de saúde coletiva.

Com base nesses dados o presente trabalho visa esclarecer por meio de uma revisão, algumas comorbidades associadas à obesidade e também demonstrar que as mesmas podem ser prevenidas com a prática regular de $\mathrm{EF}$, desde que adequadamente orientados. Fundamenta-se também, a partir deste trabalho, uma perspectiva de associar a prática da atividade física e do EF como elementos importantes na construção do debate sobre saúde coletiva e a produção científica relacionada ao fenômeno da obesidade.

\section{MATERIAL E MÉTODO}

Para a composição da presente revisão foi realizado um levantamento bibliográfico nas bases de dados Scielo, Lilacs, Pubmed, Portal de Periódicos da Coordenação de Aperfeiçoamento de Pessoal de Nível Superior (CAPES) e a busca de dados no Google Acadêmico de artigos científicos publicados até 2014 utilizando como descritores isolados ou em combinação: Obesidade, doenças cardiovasculares, saúde coletiva, exercícios físicos e adicionalmente a consulta de livros acadêmicos para complementação das informações sobre exercícios físicos como ferramenta de enfrentamento às comorbidades associadas à obesidade.

Para seleção do material, efetuaram-se três etapas. A primeira foi caracterizada pela pesquisa do material, a segunda compreendeu a leitura dos títulos e resumos dos trabalhos, visando uma maior aproximação e conhecimento, sendo excluídos os que não tivessem relação e relevância com o tema. Após essa seleção, buscaram-se os textos que se encontravam disponíveis na íntegra, totalizando 43 trabalhos, sendo estes inclusos na revisão.

Como critérios de elegibilidade e inclusão dos artigos, analisaram-se a procedência da revista e indexação, estudos que apresentassem dados referentes à obesidade e suas comorbidades, publicados entre os anos de 1990 até o mais atual 2014. Como critério de exclusão utilizou-se referência incompleta e informações presentemente desacreditadas.

\section{Comorbidades associadas à obesidade}

A obesidade é uma doença em que o excesso de gordura corporal pode atingir graus capazes de afetar a saúde. Delbim e Baciuk ${ }^{3}$, consideram a obesidade e seus fatores de risco correlacionados como um mal da sociedade contemporânea e, para tanto, incentiva investimentos que, numa abordagem regionalizada possam colaborar com a reversão de tão alarmantes dados populacionais.

Segundo Campos ${ }^{12}$, o termo "obesidade" não é o mesmo que "excesso de peso". Há uma importante diferenciação entre estes termos, argumentos e instrumentos científicos como justificativa onde o excesso de peso é definido como o peso corporal que excede o peso normal ou padrão para uma pessoa, baseado em tabelas de peso e altura, o Índice de Quetelet ou índice de massa corporal (IMC) e obesidade, situação em que o indivíduo possui uma excessiva quantidade de gordura corporal.

Ainda sob o entendimento destes apontamentos, faz-se necessário o uso de medidas complementares de composição corporal que avaliem os componentes orgânicos de forma mais acurada. É sabido que gordura visceral, ou intra-abdominal, é fator de risco no desenvolver de um estado de obesidade em indivíduos, o que não encerra em si este processo 
avaliativo quando se vislumbra um coletivo populacional, tamanha a diversidade corporal existente.

Antes da década de 80, nos EUA, as taxas de sobrepeso eram em torno de $15 \%$ e, na década de 90 , houve um salto para impressionantes $35 \%$. Hoje em dia a prevalência de obesidade e sobrepeso neste país já atingiu $65 \%$ das pessoas. Em âmbito mundial, aproximadamente $15 \%$ da população (300 milhões de pessoas) são obesas e estima-se que o Brasil, em 2025, será o quinto país a ter problemas de obesidade em seus habitantes ${ }^{13}$.

A etiologia da obesidade, por ser caracterizada como multifatorial, é de difícil interpretação, identificação e manejo. Sabe-se que a interação entre fatores ambientais, comportamentais, culturais, genéticos, fisiológicos e psicológicos são as principais causas para o ganho de peso ${ }^{14}$.

Segundo Oliveira ${ }^{15}$, os fatores que induzem a obesidade se dividem em dois grupos distintos: exógeno e endógeno. $\mathrm{Na}$ obesidade endógena os fatores que desencadeiam o processo são de ordem hormonal, já na obesidade exógena, os fatores ambientais (dieta e atividade física) figuram como determinantes. Corroborando com as afirmações de Oliveira $^{15}$, Escrivão et al. ${ }^{16}$ afirmam que mais de $95 \%$ dos casos de obesidade são devidos à fatores ambientais.

Estudos mostram que a obesidade também pode ser um fator hereditário. Num desses estudos, foi demonstrado que há perfis metabólicos desiguais para os mesmos estímulos, isto é, existe um componente genético que faz com que algumas pessoas tenham mais tendência para engordar do que outras. Em outro estudo, foi observado que, se um progenitor for obeso, este apresenta $40 \%$ de probabilidade de ter filhos obesos ou pelo menos tendência para isso e se os dois progenitores forem obesos a probabilidade de terem filhos obesos aumenta para $80 \%{ }^{17}$.

Pessoas obesas possuem chances maiores de adquirir diversas comorbidades, dentre elas, a HAS, diabetes mellitus tipo II, dislipidemias, doenças cardiovasculares, entre outras. As coexistências dessas alterações metabólicas chegam a ser sete vezes maiores nos obesos ${ }^{18}$.

No diabetes mellitus tipo II, Vancini e Lira ${ }^{19}$ descrevem essa doença como sendo uma doença endócrina caracterizada por elevada taxa de glicose sanguínea. Este fenômeno, conhecido como hiperglicemia, decorre da falta de insulina ou incapacidade da insulina em exercer adequadamente seus efeitos nos tecidos alvos.

Este fator corresponde a $90 \%$ dos casos de diabetes e ocorre geralmente em pessoas obesas com mais de 40 anos de idade, embora na atualidade se verifique com maior frequência em jovens, em virtude dos maus hábitos alimentares, sedentarismo e estresse da vida urbana.

Nesse tipo de diabetes encontra-se a presença de insulina, porém sua ação é dificultada pela obesidade, o que é conhecido como resistência insulínica, umas das causas da hiperglicemia ${ }^{19}$. Segundo Campos ${ }^{12}$, nestes casos, a insulina não consegue desempenhar com eficiência o papel de facilitar o transporte da glicose para dentro da membrana da célula.

No estudo realizado por Campbell e Gerich ${ }^{20}$, mostrou que indivíduos com IMC entre 19 à $25 \mathrm{Kg} / \mathrm{m}^{2}$ possuíam sensibilidade normal à insulina. Todavia, pessoas com IMC entre 25 à $26 \mathrm{Kg} / \mathrm{m}^{2}$, há um decréscimo da sensibilidade à insulina constatando o aumento da resistência à mesma. Por ser pouco sintomático, o diabetes permanece por muitos anos sem diagnóstico e sem tratamento, o que favorece a ocorrência de complicações no coração e cérebro.

A HAS caracteriza outra comorbidade frequente associada à obesidade, sendo importante mencionar que se trata de uma síndrome que não a encerra somente com sua relação frente a obesidade. Longo et al. $^{21}$ revelam que estudos de diferentes populações sugerem que o sobrepeso e a obesidade podem ser responsáveis por até $65 \%$ a $70 \%$ do risco de desenvolvimento de HAS.

Martelli $^{22}$ define a HAS como uma situação clínica de natureza multifatorial caracterizada por níveis de PA elevados, sendo considerada hoje uma dos principais fatores de risco para a morbidade e mortalidade cardiovascular. Associa-se a alterações funcionais e/ou estruturais dos órgãos-alvo como coração, encéfalo, rins e vasos sanguíneos e a alterações metabólicas, com consequente aumento do risco de eventos cardiovasculares fatais e não-fatais ${ }^{23}$.

Os mecanismos envolvidos na HAS induzida por obesidade são diversos e complexos. Anormalidades renais com aumento da reabsorção de sódio e água, resistência à insulina/hiperinsulinemia, hiperleptinemia, ativação do sistema reninaangiotensina e ativação do sistema nervoso simpático têm sido apontadas como mecanismos fisiopatogênicos da HAS associada à obesidade. Ao mesmo tempo têm sido relatado em pacientes obesos alterações hemodinâmicas como elevação do débito cardíaco, frequência cardíaca e volume extracelular, cujos mecanismos ainda são incertos ${ }^{24}$.

Segundo Moreira et al. ${ }^{25}$, levantamentos da OMS indicam que aproximadamente 800 milhões de pessoas tenham elevado o nível de sua pressão arterial, e bem provável que 7 milhões destas pessoas morram a cada ano, o que alarma a HAS em níveis altíssimos de correlações com outras doenças, sobretudo cardiovasculares.

Neste sentido, a perda de peso é a medida imediata no tratamento da HAS relacionada à 
obesidade, pois em geral se associa à redução da gordura visceral, causa de maior risco cardiovascular 24.

Associado à obesidade, encontramos outras doenças cardiovasculares. As principais são o infarto, o acidente vascular cerebral, arritmias cardíacas e isquemias. A principal característica das doenças cardiovasculares é a presença de aterosclerose, causada acúmulo de placas de gorduras - ateroma nas artérias, fato que impede a passagem do sangue ${ }^{26}$ e a irrigação correta dos tecidos.

A obesidade está intimamente relacionada à etiologia da dislipidemia, pois compartilham da mesma fonte de fatores de risco ${ }^{27}$ o que justifica por associação positiva entre indicadores de adiposidade com o diagnóstico de dislipidemia ${ }^{28}$.

As dislipidemias são alterações da concentração de lipídeos no sangue. Os lipídeos são responsáveis por várias funções como a produção e armazenamento de energia, absorção de vitaminas, dentre outros, mas o excesso está relacionado à aterosclerose ${ }^{29}$.

A aterosclerose é uma doença inflamatória crônica de origem multifatorial que ocorre em resposta à agressão endotelial, acometendo principalmente a camada íntima de artérias de médio e grande calibre. A formação da placa aterosclerótica inicia-se com a agressão ao endotélio vascular devida a diversos fatores de risco como dislipidemia, HAS ou tabagismo $^{30}$.

A crescente industrialização, urbanização e mecanização estão associadas com mudanças nos hábitos de vida e na dieta das pessoas. Uma dieta pobre em nutrientes e rica em gorduras e açúcares favorece o surgimento da obesidade e dos distúrbios dos lipídios ${ }^{27}$.

Diante disto, são prementes ações de combate à dislipidemia, sobretudo em adolescentes obesos que, naturalmente, são mais propensos aos desfechos clínicos negativos desta associação ${ }^{31}$.

\section{Exercícios físicos como método não farmacológico} na obesidade

O corpo humano é planejado para movimentos e atividades, inclusive de nível extenuante, embora a atividade física de intensidade leve e moderada faça parte do estilo de vida padrão. Não se pode esperar que o organismo humano apresente um funcionamento ótimo e permaneça saudável por longos períodos se ele não for adequadamente utilizado ${ }^{32}$.

Entende-se que EF é uma subcategoria de atividade física planejada, estruturada e repetitiva, e tem como objetivo melhorar ou manter um ou mais componentes da aptidão física e quando praticados com intensidade moderada e vigorosa traz benefícios à saúde $^{10}$. Weineck ${ }^{33}$ estende este entendimento à natureza humana e sua necessidade de movimentar-se por excelência existencial afirmando que todos os sistemas orgânicos e do sentido precisam ser estimulados por meio do movimento, da atividade física e do esporte para uma formação e desenvolvimento ótimo, assim como para manutenção ou aumento da capacidade de rendimento, principalmente na fase de crescimento - infância e adolescência. Este autor enriquece a pesquisa quando revela através de indicadores percentuais que a melhoria da saúde, devido ao aumento da prática de atividade física, manifesta-se em um reduzido índice de ocorrência de doenças, que é de $50 \%$ em relação à média da população para aqueles que praticam esporte.

A prática regular de EF é uma grande estratégia no combate as comorbidades associadas à obesidade. Praticar regularmente EF é de fundamental importância no processo de promoção da saúde de pessoas acima do peso, e juntamente aliado a hábitos saudáveis como uma boa alimentação, sono regular, dentre outros fatores.

Para Rocca et al. ${ }^{34}$ baseados nos estudos de Monteiro et al. "modificações na dieta associada com exercício físico favorecem a perda de massa corporal total, além de reduzir a obesidade abdominal e melhorar a tolerância à glicose."

Civinski et al. $^{36}$ relatam que os exercícios físicos atuam de forma aguda e crônica em diversas comorbidades. Exercícios resistidos ajudam no ganho e manutenção da massa muscular, fortalecendo os músculos e os exercícios aeróbios auxiliam na perda de gordura corporal, melhoram a capacidade cardiorrespiratória fortalecendo o coração e os pulmões.

Contudo, pode-se dizer que para tais benefícios, a prática dos EF deve ser regular. McArdle et al. ${ }^{2}$ afirmam que o número de sessões semanais de exercício varia de três a quatro vezes. Exercitar apenas duas vezes por semana não modifica significativamente o peso e gordura corporal. A OMS recomenda que a prática do $\mathrm{EF}$, de acordo com a faixa etária dos indivíduos seja para crianças e adolescentes: 60 minutos de atividade moderada a intensidade vigorosa por dia; para adultos $(18+) 150$ minutos de atividade de intensidade moderada por semana ${ }^{10}$.

Reforçando a afirmativa, Castro ${ }^{37}$ aponta que para promover a saúde e a perda de peso o gasto semanal em atividades físicas deva ser igual ou superior a $2000 \mathrm{kcal}$ ou de 150 a 200 minutos por semana.

Para reforçar a ideia de que o número mínimo de sessões semanais de treino deve ser de três vezes, Rocca et al. ${ }^{34}$ mostram por meio de um programa de atividade física feito com mulheres obesas, que a prática de EF sistematizado é de grande valia para diminuição do tecido adiposo e consequentemente 
para a diminuição de determinadas comorbidades associadas a obesidade.

Esse programa consistia numa prática de exercícios físicos para vinte e duas mulheres com idade média de quarenta e um anos (mínimo de 25 e máximo de 55 anos) durante três meses, três vezes por semana, com duração de uma hora, sendo trinta minutos de exercício aeróbio e trinta minutos de exercício resistido e nenhuma restrição alimentar. O Quadro 1 ilustra os resultados antes e após o programa.

Quadro 1. Medidas antropométricas e valores relativos à pressão arterial e potência aeróbia antes e após o programa $(n=22)$. Extraído e modificado de Rocca et al. ${ }^{34}$

\begin{tabular}{|c|c|c|}
\hline & Valores iniciais & Valores finais \\
\hline Peso $(\mathrm{kg})$ & 95,3 & 94,61 \\
\hline Estatura (cm) & 162,14 & 162,14 \\
\hline IMC (kg/m²) & 36,04 & 35,78 \\
\hline CC (cm) & 103,17 & 99,38 \\
\hline CQ (cm) & 123,39 & 121,41 \\
\hline RCQ & 0,84 & 0,82 \\
\hline FC repouso $(\mathbf{b p m})$ & 85,29 & 84,33 \\
\hline PAS repouso (mmHg) & 131,68 & 130,9 \\
\hline PAD repouso (mmHg) & 92,27 & 87,72 \\
\hline VO $_{2}$ Pico & 19,88 & 23,02 \\
\hline
\end{tabular}

IMC : Índice de massa corporal; CC: circunferência da cintura; CQ: circunferência do quadril; RCQ:relação cintura quadril; FC: frequência cardíaca; PAS: pressão arterial sistólica; PAD pressão arterial diastólica; $\mathrm{VO}^{2}$ : consumo de oxigênio

A análise dos resultados desse programa de treinamento revela algumas variáveis interessantes, tais como a diminuição de quase todos os itens importantes, como a gordura corporal e a PAS e PAD, sem falar no aumento de $\mathrm{VO}_{2}$, nos levando a entender a importância da prática do EF sistematizado, ressaltando que não houve restrição alimentar nessa pesquisa, sendo que, o exercício físico aliado a uma ingesta saudável de alimentos poderia apresentar dados mais benefícios e significativos para os obesos.

Silva et al. $^{38}$ afirmam que o EF sistematizado pode produzir importantes benefícios a curto, médio e longo prazo a saúde do indivíduo. Esses benefícios estão apresentados no Quadro 2.

QUADRO 2 - Benefícios associados ao exercício físico sistematizado. Extraído e modificado de Silva et al ${ }^{38}$

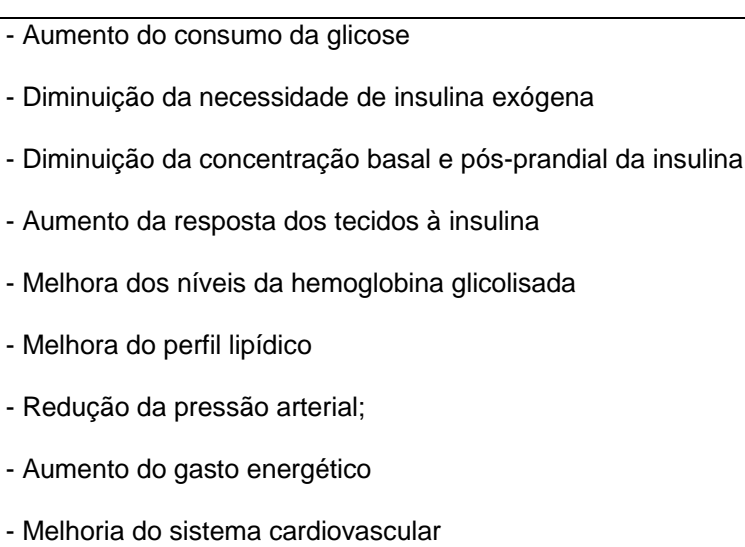

Segundo Powers e Howley ${ }^{39}$ a atividade física constitui a parte mais variável do lado do gasto energético, representando de $5 \%$ a $40 \%$ do gasto calórico total diário. A combinação de EF com restrição calórica representa um meio flexível e efetivo de conseguir uma redução ponderal. O exercício melhora a mobilização e o catabolismo de gorduras, acelerando a redução do tecido adiposo.

Robertson e Vohora $^{40}$ relatam que a atividade física bem orientada por profissionais de educação física, representa uma importante arma para melhoria da qualidade de vida de obesos ajudando sobremaneira no processo de emagrecimento. Corroborando com esta afirmativa, Jesus e Copetti ${ }^{41}$ destacam sua importância e eficácia como práticas de prevenção no combate à obesidade.

A prática regular de atividade física implica em uma transformação do indivíduo acerca de todas suas vivências diárias. Ao passo que a atividade física vai se transformando numa prática de vida do indivíduo, outras transformações dentro da rotina diária vão se evidenciando. $\mathrm{O}$ indivíduo passa vivenciar processos que operam em sinergia com a promoção em saúde e impactam positivamente em sua qualidade de vida ${ }^{42}$. Quando o indivíduo em questão é classificado como obeso, isto se evidencia de forma ainda maior.

Denadai et al. ${ }^{43}$ enfatizam este entendimento quando citam que o EF pode contribuir como modelo de intervenção apropriado para o controle precoce da obesidade e para a minimização de riscos mórbidos associados podendo ser um recurso importante para a diminuição da incidência e prevalência de obesidade.

Além dos benefícios fisiológicos, a prática de EF regulares acarreta benefícios psicológicos, pois a pessoa obesa sofre com o preconceito da sociedade, que sustenta que o corpo habitável e definido como saudável é o corpo esbelto ou magro, por assim dizer.

Em nossos tempos, define-se que uma pessoa bonita e de sucesso é magra. Isto acarreta no indivíduo obeso adversidades quanto ao enfrentamento e afirmação no mundo contemporâneo ${ }^{42}$. Sem falar que tal preconceito pode prejudicar o obeso em outras diversas situações, tais como: num relacionamento afetivo, numa entrevista de emprego e também em situações que podem ser constrangedoras.

Silva et al. $^{38}$ descrevem que os benefícios psicológicos para os obesos que praticam atividade física sistematizada são: melhor sensação de bem estar, humor e auto-estima, assim como, redução da ansiedade, tensão e depressão e reforçam afirmando que a implantação de um programa de exercícios físicos tende a aumentar o estado de saúde dos participantes, consequentemente ajudando no combate a obesidade, diminuindo a dependência, favorecendo a socialização, e com isso, melhorando sua qualidade de vida. 
Assim, entende-se que a prática regular do EF é elemento indissociável ao enfrentamento da obesidade e suas comorbidades.

\section{CONCLUSÃO}

O presente trabalho visou em seu percurso apresentar e destacar a importância de se pensar em saúde e promoção da qualidade de vida diante de toda problemática oriunda do fenômeno obesidade.

$\mathrm{Na}$ premissa de que toda atividade física é benéfica, o presente trabalho aponta que, quando o $\mathrm{EF}$ é desempenhado de maneira sistematizada sob orientação de um profissional qualificado, o indivíduo obeso de fato se beneficia. Indicadores estatísticos sugerem que, considerando o cenário atual no qual a obesidade ganha contornos de pandemia, a prática de EF sistematizado contribui em todos os âmbitos para o enfrentamento e combate à doença e comorbidades associadas.

O EF sistematizado é visto como uma possibilidade de transformação de todo um modo de viver em sociedade, podendo trazer melhorias $\mathrm{e}$ entendimentos ao campo da saúde pública.

O enfrentamento da obesidade e comorbidades oriundas pela via do EF contribuem como melhorias no aspecto psicológico e não apenas do indivíduo, ou indivíduos classificados como obesos, mas também de todo seu ambiente ao redor.

Acerca de todo material estatístico oriundo da literatura pesquisada, o presente trabalho sugere que a grande contribuição do EF sistematizado seja mais do que seu comprovado benefício corporal a quem faz desta prática uma excelência de vida, porém, salientamos que o profissional da educação física é de grande importância, seja na orientação dos exercícios, como também no processo de emagrecimento.

As discussões e os estudos devem continuar sobre o referido tema de modo a suplantar hiatos não atendidos pelo presente trabalho.

\section{REFERÊNCIAS}

1. Stadler TAC, Moretti M, Marcelo P, Moretti MP, Sakae TM, Araujo D. Associação dos níveis de dislipidemia entre obesidade tipo I, II e III. Arq Cat Med. 2011;40(3):21-4.

2. Mcardle WD, Katch FI, Katch VL. Fisiologia do Exercício: Energia, Nutrição e Desempenho Humano. 5. ed. Rio de Janeiro: Guanabara Koogan; 2003.

3. Delbim LR, Baciuk EP. Sobrepeso, obesidade e ônus urbano: projeções pandêmicas ou sensacionalismo? Intellectus. Rev Acadêmica Digital das Faculdades Unopec. 2012;8(2):28-43.

4. Freedman DS, Khan LK, Dietz WH, Srinivasan SR, Berenson GS. Relationship of childhood obesity to coronary heart disease risk factors in adulthood: The Bogalusa Heart Study. Pediatrics. 2001;108(3):712-8.

5. Fujimoto WY, Bergstron RW, Boyko DJ, Chen KW, Leonetti D, Newell-Morris L, Shofer JB, Wahl PW. Visceral adiposity and incident coronary heart disease in Japanese-American men. Diabetes Care. 1999; 22(11):1808-12.

6. Heyward VH, Stolarczyk LM. Avaliação da composição corporal aplicada. São Paulo: Manole, 2000.

7. Lean ME, Han TS, Seidell JC. Impairment of health and quality of life in people with large waist circumference. Lancet 1998; 351(9106):853-6.

8. Rexrode KM, Carey VJ, Hennekens $\mathrm{CH}$, Walters EE, Colditz GA, Stampfer MJ, Willett WC, Manson JE. Abdominal adiposity and coronary heart disease in women. JAMA. 1998; 280(21):1843-8.

9. Cercato C, Silva S, Sato A, Mancini M, Halpern A. Risco cardiovascular em uma população de obesos. Arq. Bras Endocrinol Metab. 2000; 44(1):45-8.

10. World Health Organization. Obesity and overweight. Acesso em 4 de Março de 2014. Disponível em: http://www.who.int/mediacentre/ factsheets/fs311/en/

11. Matsudo SM, Matsudo V, Rodrigues VK, Mahecha SM. Atividade física no tratamento da obesidade. Einsten. 2006;4(Supl.1):29-43.

12. Campos MA. Musculação: diabéticos, osteoporóticos, idosos, crianças, obesos. Rio de Janeiro: Sprint; 2000.

13. Neto AS, Saheb GCB, Arantes BS, Júnior JP, Silva RBS, Nara AB. Fatores ambientais e neuroendocrinológicos envolvidos na gênese da epidemia da obesidade. Arq. Bras Ciências de saúde. 2007;33(1):44-53.

14. Andrade DR, Magalhães FR. Prevalência da síndrome metabólica em pacientes de consultório. Revista Brasileira de Obesidade, Nutrição e Emagrecimento. 2009;3(17):448-54.

15. Oliveira RJ. Saúde e Atividade Física: Algumas Abordagens Sobre Atividade Física Relacionada à Saúde. Rio de Janeiro: Shape; 2005.

16. Escrivão MAMS, Oliveira FLC, Taddei JAAC, Lopes FA. Obesidade exógena na infância e na adolescência. J Pediatr. 2000;76(3):305-10.

17. Póvoas F. O Prazer de Emagrecer. $6^{\circ}$ ed. Porto: Caderno; 2007.

18. Lavrador MSF. Riscos cardiovasculares em adolescentes com diferentes graus de obesidade. Arq Bras Cardiol. 2010;96(3):205-11.

19. Vancini RL, Lira CAB. Aspectos gerais do diabetes mellitus e exercício. Centro de Estudo de 
Fisiologia do Exercício, 2004.

20. Campbell PJ, Gerich JE. Impact of obesity on insulin action in volunteers with normal glucose tolerance: demonstration of a threshold for the adverse effect of obesity. J Clin Endocrinol Metab. 1990;70(4):1114-8.

21. Longo MAT, Martelli A, Zimmermann A. Hipertensão Arterial Sistêmica: aspectos clínicos e análise farmacológica no tratamento dos pacientes de um setor de psicogeriatria do Instituto Bairral de Psiquiatria, no Município de Itapira, SP. Rev. Bras Geriatr Gerontol. 2011;14(2):271-84.

22. Martelli A. Potencial da pratica de exercícios físicos regulares como método não farmacológico no controle da Hipertensão Arterial Sistêmica. Desenvolvimento Pessoal 2013;3(1/2):39-51

23. SBC - Sociedade Brasileira de Cardiologia. VI Diretrizes Brasileiras de Hipertensão. Arq Bras Cardiol. 2010:95(1supl.1):1-51.

24. Galvão R, Kohlmann Jr O. Hipertensão arterial no paciente obeso. Rev Bras Hipertens 2002;9(3):262-7.

25. Moreira NF, Muraro AP, Brito FSB, GonçalvesSilva RMV, Sichieri R, Ferreira MG. Obesidade: Principal fator de risco para hipertensão arterial sistêmica em adolescentes brasileiros participantes de um estudo de coorte. Arq Bras Endocrinol Metab. 2013;57(7):520-6.

26. Ministério da Saúde. Doenças cardiovasculares causam quase 30\% das mortes no País - Set. 2011. Disponível em: http://www.brasil.gov.br/ saude/2011/09/doencas-cardiovasculares-causamquase-30-das-mortes-no-pais. Acesso em: novembro 2015.

27. Howard BV, Ruotolo G, Robbins DC. Obesity and dyslipidemia. Endocrinol Metab Clin North Am. 2003;32(4):855-67.

28. Mancini MC. Obesidade e Doenças Associadas. In: Mancini MC, Geloneze B, Salles JEN, Lima JG, Carra MK. Tratado de Obesidade. Itapevi: AC Farmacêutica, 2010.

29. Conselho Federal De Nutrição. Dislipidemia - O que o Nutricionista faz por você? Disponível em: http://www.cfn.org.br/_eficiente/repositorio/ Comunicacao/ Material_ institucional/ 167.pdf. Acesso em: Mar. 2013.

30. Sociedade Brasileira De Cardiologia. V Diretriz Brasileira de Dislipidemias e Prevenção da Aterosclerose. Arq Bras Cardiol. 2013;101(4) supl.1:1-20.

31. Lira CTC, Cardoso Júnior CG, Gomes PP, Tenório TRS, Prado MCL, Ferreira MNL, et al. Efeitos de diferentes intensidades de treinamento aeróbio sobre a lipemia de adolescentes obesos. Rev Bras Ativ Fis e Saúde. 2013;18(6):761-70.
32. Martelli A. Aspectos fisiopatológicos da aterosclerose e a atividade física regular como método não farmacológico no seu controle. Saúde e Desenvolvimento Humano 2014;2(1):41-52.

33. Weineck J. Atividade Física e esporte para quê? 7 ed. São Paulo: Manole; 2003.

34. Rocca SVS, Tirapegui J, Melo CM, Ribeiro SML. Efeito do exercício físico nos fatores de risco de doenças crônicas em mulheres obesas. Revista Bras Cienc Farmac. 2008;44(2):185-92.

35. Monteiro RCA, Riether PTA, Burini RC. Efeitos de um programa misto de intervenção nutricional e exercício físico sobre a composição corporal e os hábitos alimentares de mulheres obesas em climatério. Rev Nutr 2004;17(4):479-89.

36. Civinski C, Montibeller A, Braz ALO. A importância do exercício físico no envelhecimento. Revista da Unifebe. 2011;9(1):163-75.

37. Castro ACB. O impacto do sedentarismo na qualidade de vida dos trabalhadores e sua consequência para as organizações [monografia]. Rio de Janeiro: Universidade Cândido Mendes; 2011.

38. Silva AG, Rodrigues VD, Neto ATM, Machado LF. Atividade física na promoção da saúde. Revista digital, Buenos Aires 2010. Acesso nov 2013. Disponível em: www.edfdeportes.com/ efd145/ atividade-fisica-na-promocao-da-saude. htm

39. Powers SK, Howley ET. Fisiologia do Exercício: Teoria e Aplicação ao Condicionamento Físico e ao Desempenho. 3. ed. São Paulo: Manole; 2000.

40. Robertson N, Vohora R. Fitness vs. Fatness: Implicit Bias Towards Obesity Among Fitness Professionals and Regular Exercises. School of Psychology, University of Leicester, UK, 2007.

41. Jesus RF, Copetti J. Conhecimentos teóricos sobre atividade física e obesidade em estudantes de educação física de uma universidade privada. Rev Educ Fis Unicamp. 2013;11(4):110-11.

42. Zambelli CL. A academia de ginástica, seus frequentadores e discursos: experiências no corpo e com o corpo[ trabalho de conclusão do curso].Araras: Centro Universitário Hermínio Ometto, 2010.

43. Denadai RC, Vítolo MR, Macedo AS, Teixeira L, Cezar C, Dâmaso AR, Fisbeg GM. Efeitos do exercício moderado e da orientação nutricional sobre a composição corporal de adolescentes obesos avaliados por densitometria óssea (DEXA). Rev Paul Fís. 1998;12(2):210-8. 
Arch Health Invest (2016) 5(2): 112-119

\section{CONFLITO DE INTERESSES}

Os autores declaram não haver conflitos de interesse.

\section{AUTOR PARA CORRESPONDÊNCIA}

Anderson Martelli

martellibio@hotmail.com

Submetido em 28/03/2016

Aceito em 08/04/2016 